\title{
Skén\&graphie
}

SKIINagraphil: Coulisses des arts du spectacle et des scènes

émergentes

$6 \mid 2019$

Voyages des cultures et des mémoires dans les arts de la scène

\section{Verteidigungsrede des Somalischen Piraten - Défense du pirate somalien}

Extrait

Wolfram Lotz

Traducteur : Claire Carnin

\section{OpenEdition}

\section{Journals}

Édition électronique

URL : https://journals.openedition.org/skenegraphie/3018

DOI : 10.4000/skenegraphie.3018

ISSN : 2553-1875

Éditeur

Presses universitaires de Franche-Comté

\section{Édition imprimée}

Date de publication : 1 novembre 2019

Pagination : 193-198

ISBN : 978-2-84867-673-9

ISSN : 1150-594X

Référence électronique

Wolfram Lotz, « Verteidigungsrede des Somalischen Piraten - Défense du pirate somalien », Skén\&graphie [En ligne], 6| 2019, mis en ligne le 01 juin 2021, consulté le 27 septembre 2021. URL : http://

journals.openedition.org/skenegraphie/3018; DOI : https://doi.org/10.4000/skenegraphie.3018

Ce document a été généré automatiquement le 27 septembre 2021.

Presses universitaires de Franche-Comté 


\title{
Verteidigungsrede des Somalischen Piraten - Défense du pirate somalien
}

\section{Extrait}

\author{
Wolfram Lotz \\ Traduction : Claire Carnin
}

Monsieur le juge, mon nom est Ultimo Michael Foufoun, et comme vous le savez et comme d'ailleurs chacun pouvait l'apprendre dans la presse allemande je suis un petit nègre de Somalie. Pour plus de commodité je m'adresse à vous en allemand. Je compte sur votre compréhension, car tout s'en trouvera grandement facilité selon moi. En ce qui concerne ma personne, je ferai les déclarations suivantes : j'ai vu le jour pendant la saison des pluies, sous un arbre. Ses fleurs embaumaient le matin plus que les autres fleurs de tous les autres arbres et quand venait le midi ses corolles craintives se fermaient aux regards des hommes et des bêtes. Le nom de mon père était ordinairement Kanok; parfois il s'appelait aussi Pulpin, parfois il s'appelait Thoarde, parfois il se nommait Iltis, c'était en fonction de l'interlocuteur. De profession, il faisait partie de ces gens à la périphérie de Mogadiscio qui fouillent le sable avec un bâton en quête d'objets en tous genres. Ma mère s'appelait Honija, elle avait appris toutes sortes de métiers: faire cuire des choses dans son faitout en fer-blanc, laver la lessive, astiquer nos menus effets domestiques, pleurer de chagrin, chanter de gaité, programmer des tableaux de base dans Excel, houspiller les chiens avec une branche de bois mort quand ils se faisaient trop envahissants, insuffler un sentiment de sécurité. Je prends acte que je comparais ici devant le tribunal de grande instance de Hambourg pour délit de piraterie. C'est vrai, je suis un pirate. Je reconnais également avoir commis le fait qui m'a été reproché, l'assaut du cargo MS Taipan. J'ai toutefois le droit d'exposer comment j'en suis venu à perpétrer cet acte et voudrais ce faisant vous éclairer sur la situation qui nous y a conduit, aussi étranges et incompréhensibles nos conditions de vie puissent-elles sembler, à vous autres Européens. Mais donc : avant de m'adonner à la piraterie, j'ai commencé par apprendre la pêche. J'ai connu ce métier grâce à mon meilleur ami Tofdau. Depuis tout petit, Tofdau voulait devenir pêcheur. Moi tout petit ça m'a toujours été complètement égal ce que j'allais faire plus tard. 
Quand j'étais petit, je disais toujours : ça m'est complètement égal ce que je ferai plus tard et vous pouvez tous aller vous faire huiler le fion. Pourtant, le soir, quand nous nous retrouvions à deux avec Tofdau dans une barrique de tôle éventrée derrière une vieille usine à la périphérie de Mogadiscio, qui fut, jadis, une fabrique de bananes à l'époque coloniale, quand nous nous retrouvions donc le soir dans cette barrique à l'arrière de l'usine, Tofdau me racontait tout ce qu'il savait de la pêche. À vrai dire, ce n'était pas beaucoup, mais c'était beau et plein de passion. Et là, un jour, moi aussi j'ai commencé à rêver de devenir pêcheur et Tofdau et moi nous sommes fait le serment de devenir pêcheurs ensembles. Tofdau disait : «Quand on sort de la ville et qu'on regarde la mer, on peut voir là- bas une foule bigarrée de bateaux. Ce sont des navires anglais, hollandais, japonais, indiens, américains, allemands et chinois, et ils sont là parce que la mer de Somalie regorge de poissons succulents, comme le ciel regorge d'étoiles. Tout ce que nous avons à faire, c'est prendre le large et saisir notre part du trésor ».

2 Ainsi, quand vint le temps, quand une force nouvelle eut envahi nos corps et que des poils nous furent poussés sous les bras, Tofdau et moi avons noué des filets et nous nous sommes payés avec l'argent que Tofdau avait hérité de sa grosse maman,

et avec l'argent qu'il tirait d'on ne sait trop où (personne ne sait rien de précis à ce sujet) et avec l'argent que je m'étais fait en achetant un poste de radio HS, en le réparant et en le revendant sur internet,

et avec l'argent que j'avais touché en construisant un petit muret de jardin, et avec l'argent que j'avais gagné en vendant un oiseau au plumage bleuté que j'ai capturé à la main derrière chez le vieil Olim, et avec l'argent que j'avais touché en vendant une cassette de rap français, et avec l'argent que j'avais gagné en forant un petit fossé entre deux autres fossés forés par d'autres personnes à des fins d'irrigation, avec cet argent nous nous sommes payé un petit bateau cabossé. Et nous l'avons appelé L'Espoir. ( $\mathrm{Si}$ jusqu'ici il y a des questions, surtout: allez-y. Si quoi que ce soit ne vous semble pas concluant, il suffit de demander. Bien.) Et puis enfin, le grand jour arriva : nous avons pris la mer sur notre bateau. Quand les côtes furent à plusieurs kilomètres, Tofdau a coupé le moteur et nous avons écouté le silence. Silence. Ensuite ce furent des embrassades, des rires, on a laissé glisser nos filets dans l'eau. Au bout d'un moment, on a remonté les filets. Mais on ne pouvait pas en croire nos yeux, car nos filets étaient vides. Alors on s'est penché vers la mer, on a regardé et on a vu que la mer aussi, elle était toute vide. Les Anglais, les Hollandais, les Japonais, les Indiens, les Américains, les Allemands, les Chinois, avec leurs navires de pêche, ils avaient pêché tous les poissons et tout le reste aussi. Et la mer était toute vide, et il n'y même avait plus une seule algue, même plus le moindre plancton, rien, rien, il n'y avait même plus d'eau. La mer était si claire qu'on pouvait voir au-travers, de plus en plus bas, jusqu'au fond, rien, rien, pas une goutte de flotte et rien d'autre non plus, elle était si vide la mer, et là, tout en bas, tout en bas au fond, Tofdau et moi on a vu briller le fond de la mer, mais il n'était pas de sable comme nous l'avions toujours cru, mais de rage, immense et sans fin comme la rage. Alors Tofdau s'est mis à crier, il hurlait comme une bête blessée et voulait se précipiter dans ce vide mais moi je l'ai agrippé, je me suis cramponné à lui comme une mère se cramponne à son gosse lorsque les milices arrivent, je me suis cramponné à mon ami Tofdau pour qu'il ne puisse pas sauter, je me suis cramponné de toutes mes forces.

On a rejoint la terre ferme à la nuit tombée. Tofdau est descendu du bateau sans un mot et il s'est évanoui dans l'obscurité. J'étais liquéfié. J'ai mis notre bateau sous une bâche 
et j'ai arpenté la ville plongée dans la nuit, la tête chargée de pensées confuses. J’ai fini par me hisser dans un sorbier. La ville était très calme cette nuit- là, juste de temps en temps le feu lointain d'une mitrailleuse, et quand le jour pointa, ma décision était prise. Assis dans ce sorbier, je décidai de préparer mon diplôme de piraterie à la faculté de Mogadiscio. J'ai déposé plusieurs demandes de subventions et obtenu une bourse mensuelle auprès du CROUS islamiste de Mogadiscio, une petite pension du Ministère de l'Enseignement supérieur et de la Recherche somalien ainsi que d'autres allocations au mérite allouées par la Fondation pour la formation professionnelle d'Afrique de l'Est. Durant ma Licence et mon Master, j'ai étudié l'abordage de bâtiments d'époque et actuels, la chasse furtive d'un cargo par nuit noire, la salve de mitrailleuse en signe de liesse ou pour intimider l'opposant ainsi que le cri à pleine gorge pour forcer l'équipage adverse à la reddition. J'ai décroché mon diplôme avec une très bonne mention.

Je n'avais plus revu Tofdau depuis cette fameuse nuit, mais après être passé au secrétariat des études retirer mon diplôme, je me suis lancé à sa recherche. Car dans notre culture l'amitié compte beaucoup (il faut le savoir pour pouvoir nous comprendre). J'ai donc rentré Tofdau sur Google et j'ai rapidement trouvé qu'entretemps, il s'était fait embaucher comme employé d'une crêperie d'un quartier pauvre de la ville. En allant voir sur place le lendemain, j'ai constaté que le snack en question était plutôt un morceau de tôle ondulée monté sur quatre poteaux. Tofdau y faisait des crêpes pour le compte du propriétaire éthiopien. La boîte avait l'air de bien tourner dans ce quartier, pourtant au travail Tofdau semblait malheureux comme tout. Je me suis approché, je l'ai pris dans mes bras, et je lui ai dit : « Tofdau, je n'ai plus que toi au monde - je suis pirate maintenant et je vais prendre la mer sur notre petit bateau. Je veux que tu viennes avec moi ! » Les yeux de Tofdau se remplirent de larmes, il prit la crêpe qu'il venait de cuire, la tartina avec une crème de céleri, la roula et la tendit au client affamé - puis sans attendre la monnaie il jeta son tablier dans la poussière et m'emboîta le pas. On a arpenté les rues de Mogadiscio en chantant et on est descendu jusqu'au rivage où caché sous une bâche se tenait encore notre bateau cabossé, L'Espoir. Pendant que nous attendions les navires au large des côtes (on a attendu là pendant des jours et des nuits), Tofdau m'a raconté ce qu'il avait fait durant tout le temps de mes études. Voilà ce qu'il me dit :

7 «Ultimo, mon ami, au début j'étais tellement abattu que je n'étais pas en mesure de rebondir. Je vivais au jour le jour ; je faisais une grosse dépression. Mais j'ai vite été à sec et impossible de trouver du boulot aussi rapidement. Comme je n'avais pas de solution, j'ai commencé pour de l'argent à me laisser mettre des objets dans le cul. Je n'avais encore jamais raconté ça à personne, Ultimo, tu es le premier à qui je le raconte, parce que tu es mon ami. Je me suis laissé mettre un déodorant à bille, une pince à saucisses de barbecue, un réveil radio-piloté, je me suis laissé mettre une nectarine dans le cul, l'accoudoir d'une chaise pliante de jardin, une anthologie de poésie islandaise, un chargeur vide de kalachnikov, une boîte de pâtée pour chats, le tiroir d'une table de chevet et que sais-je encore. Je me suis fait beaucoup d'argent comme ça, crois-moi, on peut vraiment gagner beaucoup avec ça, mais ce n'est pas bien. Je ne dis pas que c'est mal de se faire mettre des objets dans le cul, ça peut être une bonne et belle chose pour peu qu'on le désire. Mais si on ne fait ça que pour l'argent et sans vraiment le désirer, alors ce n'est pas bien. À force, Ultimo, on tue le petit oiseau chanteur qui niche au fond de nos cœurs. Je suis peut-être réac', mais c'est comme ça que je vois les choses. Enfin bon, après quelques mois, j'ai trouvé ce boulot à la crêperie, un bon travail comme il faut. Mais on bosse toute la journée et ça paie si mal qu'on 
n'arrive même pas à en vivre. Alors faut faire quoi, hein ? Faut faire quoi dans ce monde de chien?»

Tout à coup mon ami Tofdau fut interrompu par un bruit assourdissant, vous savez, ça faisait un peu comme : Ultimo émet un bruit de corne avec sa bouche. Vous avez reconnu? Non? Attendez!

Ultimo émet le même bruit à nouveau. Alors? Toujours pas? Attendez, je le refais: On entend une corne de brume. Vous avez reconnu maintenant? Une corne de brume! C'était une corne de brume! Vous voyez! Tout à coup un gros navire était là et il se dirigeait droit sur notre petit bateau. Nous avions manqué de vigilance et avec le soir, on ne l'avait tout simplement pas vu venir. Il était déjà trop tard pour éviter le cargo, il ne nous restait qu'une solution: l'abordage. J'avoue, je ne dirai pas que j'ai abordé ce navire uniquement pour nous sauver, $\mathrm{j}$ 'avais aussi dans l'idée de prendre le cargo d'assaut, mais ce n'est pas non plus comme si j'avais voulu aborder le bateau uniquement pour le prendre d'assaut, en fait sur le coup l'envie de prendre le navire d'assaut et l'envie de nous sauver n'étaient pas deux choses distinctes. Vous voyez ? (C'est quand même un point important!) Et donc, je disais : pile au moment où le cargo allait nous rentrer dedans, nous nous sommes lancés à l'abordage. Ici je voudrais pouvoir compter sur votre compréhension, car il m'est impossible de décrire le déroulement exact des opérations, nous autres, pirates somaliens, sommes tenus au secret par notre code de l'honneur. Ce qu'on peut dire tout de même, c'est que c'est une manœuvre presque invraisemblable et très hasardeuse qui nécessite certaines compétences. C'est d'ailleurs ce qui nous a perdus : Tofdau n'était pas entraîné, il est tombé à l'eau pendant la manœuvre. Je ne m'en suis aperçu qu'une fois sur le pont: je me suis retourné et Tofdau n'était plus là. J'ai regardé en bas, on ne voyait que les débris de notre petit bateau, ballottés par la mer déchaînée, mais pas de Tofdau. Alors mon cœur s'est glacé d'effroi parce que je n'avais pas suffisamment veillé sur mon ami. J'ai couru sur le pont et je me suis mis à crier «il faut arrêter le navire! » pas de réponse, je me suis précipité à travers les couloirs et j'ai grimpé les escaliers de l'immense bateau quatre à quatre jusqu'à la passerelle mais il n'y avait personne et j'ai crié le plus fort possible mais là encore pas de réponse. Et voilà que j'étais tout seul sur la passerelle face au poste de commande, sans connaître ni le matériel ni les logiciels on n'avait pas dû les étudier en cours (ou alors j'étais absent) - un appareil inconnu de chez inconnu et je n'avais aucune idée de comment stopper le navire. J'ai appelé à l'aide jusqu'à ce que mon cœur soit totalement paralysé par l'angoisse. Et puis la paralysie de mon cœur s'est déversée dans mon corps et j'ai perdu connaissance.

Quand je suis revenu à moi, je me suis retrouvé les poings menottés, seul sur le pont d'un bâtiment de la marine hollandaise, avec la nuit tout autour. Comme vous le savez et comme j'ai moi-même fini par l'apprendre dans les médias, l'équipage du cargo s'était en fait réfugié dans une salle de confinement et de là avait lancé un appel radio de détresse. Suite à quoi une unité spéciale de la marine hollandaise avait dû aborder le cargo et (du moins, si l'on en croit les journaux) avait libéré l'équipage ce qui revient à dire qu'elle m'avait fait prisonnier alors que je gisais sur la passerelle totalement inconscient.

11 Et dans cette vedette de la marine hollandaise qui filait sur la mer, avec au-dessus de moi le ciel nocturne et ses millions de millions d'étoiles, j'ai repensé à une histoire de ma grand-mère Kröka sur la création du ciel qu'elle nous avait raconté un jour, à mon ami Tofdau et à moi, quand nous étions encore tout gamins : 
12 « Au commencement, tout était plongé dans les ténèbres et le rien, et Dieu dit: des ténèbres et du rien, voilà qui n'est pas suffisant. Et Dieu créa une grosse mitrailleuse et il créa un pick-up et il créa une usine allemande de balles traçantes et il monta la mitrailleuse à l'arrière du pick-up et il tira comme un fou toutes les balles traçantes dans les ténèbres et c'est ainsi qu'est né le ciel ». Et là, assis sur le pont du bateau de la marine, regardant la mer et le ciel au loin, j'ai senti un effroi violent s'emparer de moi et j'ai pleuré comme jamais je n'avais pleuré jusqu'alors.

13 Voilà mon histoire. Je vous demande de me croire, je sais que ma réalité quotidienne pour vous autres Européens pourra sembler étrange et même incompréhensible, mais je vous le demande, il faut me comprendre et me croire, autant que vous le pourrez.

14 Mon avocat a eu la gentillesse de me permettre d'apporter de chez moi quelques pièces à conviction pour étayer mon histoire : qu'il en soit vivement remercié. Je voudrais vous les présenter rapidement. Tout d'abord, l'entrée de la faculté de Mogadiscio où j'ai étudié la piraterie. Ici, regardez :

15 On apporte le portail d'entrée. J'ai aussi avec moi le morceau de rivage où L'Espoir, notre bateau, était stocké sous une bâche. Regardez : On apporte le morceau de rivage. Et voici la rue de Mogadiscio où j'ai grandi. On apporte la rue. Je sais que ces preuves pourront sembler ridicules, seulement je n'en ai pas d'autres. Je sais que mon histoire aussi pourra peut-être paraître ridicule, parce que tout vous semblera étrange et exotique, mais je compte sur votre compréhension. Je compte vraiment sur votre compréhension! C'est mon unique chance. 